Signal \& Image Processing : An International Journal (SIPIJ) Vol.6, No.6, December 2015

\title{
A COMPARATIVE STUDY OF DOA ESTIMATION ALGORITHMS WITH APPLICATION TO TRACKING USING KALMAN FILTER
}

\author{
Venu Madhava $\mathrm{M}^{1}$, Jagadeesha $\mathrm{S} \mathrm{N}^{1}$, and Yerriswamy $\mathrm{T}^{2}$ \\ ${ }^{1}$ Department of Computer Science and Engineering, \\ JNN College of Engineering, Shimoga \\ ${ }^{2}$ Department of Computer Science and Engineering, \\ KLE Institute of Technology, Hubli
}

\begin{abstract}
Tracking the Direction of Arrival (DOA) Estimation of a moving source is an important and challenging task in the field of navigation, RADAR, SONAR, Wireless Sensor Networks (WSNs) etc. Tracking is carried out starting from the estimation of DOA, considering the estimated DOA as an initial value, the Kalman Filter $(K F)$ algorithm is used to track the moving source based on the motion model which governs the motion of the source. This comparative study deals with analysis, significance of Non-coherent, Narrowband DOA (Direction of Arrival) Estimation Algorithms in perception to tracking. The DOA estimation algorithms Multiple Signal Classification (MUSIC), Root-MUSIC\& Estimation of Signal Parameters via Rotational Invariance Technique (ESPRIT) are considered for the purpose of the study, a comparison in terms of optimality with respect to Signal to Noise Ratio (SNR), number of snapshots and number of Antenna elements used and Computational complexity is drawn between the chosen algorithms resulting in an optimum DOA estimate. The optimum DOA Estimate is taken as an initial value for the Kalman filter tracking algorithm. The Kalman filter algorithm is used to track the optimum DOA Estimate.
\end{abstract}

\section{KEYWORDS}

Direction of arrival (DOA), MUSIC, Root-MUSIC, ESPRIT, Tracking, Kalman filter.

\section{INTRODUCTION}

The Estimation of Direction of Arrival (DOA) and its tracking, is the most significant area of array signal processing and finds its applications in the fields of RADAR, SONAR, Wireless Sensor Networks (WSN),Seismology etc. [1]. Tracking the DOA Estimation is estimating the value of DOA of the signals from various sources impinging on the array of sensors at each scanning instant of time [2]. The tracking is performed in order to get correlated estimates at different instants of time. The correlation between the data is also known as data association or estimate association. In the first step, the plane wave fronts from far field are considered to be falling on the Uniform Linear Array (ULA) [11]. A particular number of snapshots are collected and the DOA is estimated using techniques like Multiple SIgnal Classification (MUSIC), RootMUSIC and Estimation of Signal Parameters via Rotational Invariance Technique (ESPRIT). All the three algorithms estimate the DOA of the sources which are stationary but the estimation of 
Signal \& Image Processing : An International Journal (SIPIJ) Vol.6, No.6, December 2015

DOA of the moving source is an important problem. In order to estimate the DOA of the moving target, the Estimated DOA will Act as an initial value to the Kalman filter algorithm and the Kalman filter algorithm tracks the DOA at each scanning instant of time based on the target motion model [2] [16].Unlike, this method ,MUSIC, Root-MUSIC or ESPRIT can be used to estimate the instantaneous DOA estimate provided that there is no requirement of data association and the process will be slow. In the present study, we draw brief comparisons among the three most used DOA estimation algorithms viz MUSIC [3], Root-MUSIC [4], ESPRIT [5]. These algorithms are also known as high resolution DOA Estimation algorithms. It is assumed that the Signals are non-coherent, narrowband sources. The DOA Estimation is performed in multiple source scenarios and tracking is performed on single source.

\section{BACKGROUND AND FRAMEWORK}

Estimating and tracking the signal parameters viz Time, Frequency, Phase and DOA are interesting and find applications in areas of RADAR, SONAR, Seismology, Air Traffic Control etc. There are various types of estimation techniques such as classical techniques, Beam forming, Spectral based and parametric approaches[6][13].

The Maximum Likelihood (ML) DOA estimation technique [10] [13] was originally developed by R. A. Fisher in 1920's. Under the suitable assumptions, it estimates the DOA of the incoming signal with the help of maximizing the log-likelihood function of the sampled data sequences coming from a direction.

In the beamforming technique [13], the array is steered in one direction and the output power is measured. We observe maximum power when steered direction and DOA of signal are in line. These techniques find out the output of the array by linear combination of the data received with a weight vector. If the array weight vector is used then it is known as conventional beam forming technique.

In subspace based techniques for DOA Estimation such as MUSIC [3], we get a spectrum like function of interested parameters, whose distinct peaks are the interested estimated parameters. Although MUSIC algorithm being robust and computationally less complex, it needs a search algorithm to identify the largest of the peaks. In Root-MUSIC [4] \& ESPRIT [5], a search over all the parameters of interest is carried out to get more accurate estimates being computationally expensive. In order to track the parameters, we have adaptive algorithms. These are in turn divided mainly into two types; Least Mean Square (LMS)[2][6] are the types of algorithms which converge at slow rates dependent on the number of step sizes and Recursive Least Squares (RLS)[2][6] are the types of algorithms which converge much quickly compared to the former type of algorithms. In the present literature, one of the later (RLS) type of algorithm viz Kalman filter is used to track the DOA Estimation of the moving target. The Kalman Filter algorithms proposed by R.E.Kalman in 1960 [16] are basic type of tracking algorithms which consider the state-space model of the moving target to estimate the components of motion. This section gives out the necessary framework to perform the tracking operation of the optimum DOA Estimate.

\subsection{System model}

Let us consider an ULA of ' $M$ ' identical sensors on which ' $N$ ' narrow band signals are being impinged from the directions $\theta_{1}, \theta_{2}, \theta_{3}, \ldots \ldots \ldots \theta_{N}$. The $i^{\text {th }}$ signal impinging on the array as shown in 
Signal \& Image Processing : An International Journal (SIPIJ) Vol.6, No.6, December 2015

the Fig 1 is given by

$$
S_{i}(t)=S_{i} e^{j\left(\omega t+\phi_{i}\right)} \quad i=1,2,3, \ldots, N
$$

Where, $S_{i}, \omega, \phi_{i}$ are the amplitude, frequency and phase of the signals of three parameters, phase $\phi$ is considered to be a uniformly distributed random variable. Let us define a column vector $S(t)$ as

$$
S(t)=\left[S_{1}(t), S_{2}(t), \ldots \ldots \ldots S_{N}(t)\right]^{T}
$$

Where ' $T$ ' denotes Transpose.

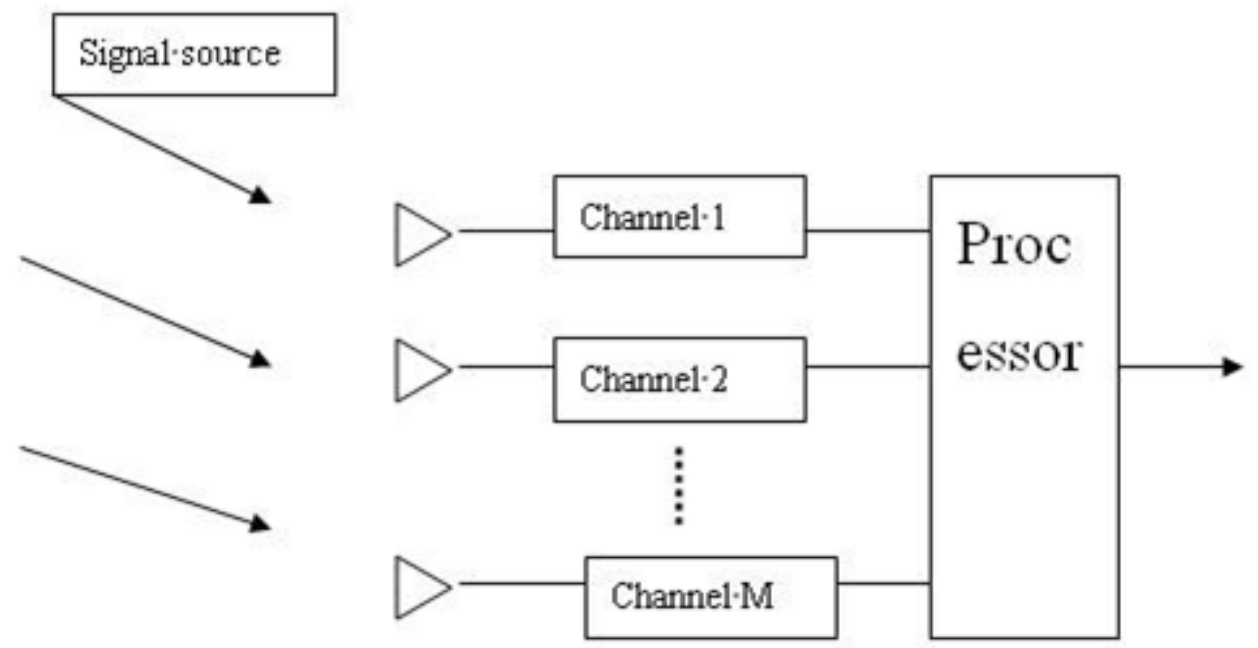

Fig 1: Illustration of the DOA Estimation Model

The direction vector of the $i^{\text {th }}$ source is given by

$$
\begin{gathered}
a\left(\theta_{i}\right)=\left[1, e^{-j \omega_{i} \tau_{1}\left(\theta_{i}\right)}, e^{-j \omega_{i} \tau_{2}\left(\theta_{i}\right)}, \ldots \ldots \ldots \ldots, e^{-j \omega_{(M-1)} \tau_{(M-1)}\left(\theta_{i}\right)}\right]^{T} \quad i=1,2,3 \ldots, N \\
\text { Where, } \tau_{m}=(m-1) \frac{d}{c} \sin \theta_{i} \quad m=1,2,3 \ldots M
\end{gathered}
$$

$d$ is the inter element spacing, $c$ being the propagation velocity of the plane wave front. The inter element spacing is assumed to be less than or equal to half the wavelength of the signal impinging. This assumption is made in order to avoid spatial aliasing.

Substitute (4) in (3) and replacing $\omega=2 \pi f_{c}$, we get the array response vector for the ULA and it is given by

$$
a\left(\theta_{i}\right)=\left[1, e^{-j \frac{2 \pi}{\lambda_{\omega}} d \sin \left(\theta_{i}\right)}, \ldots \ldots \ldots \ldots, e^{-j \frac{2 \pi}{\lambda_{\omega}}(M-1) d \sin \left(\theta_{i}\right)}\right]^{T} \quad i=1,2,3 \ldots, N
$$

Where, $f_{c}$ is the carrier frequency and $\lambda_{\omega}$ is the wavelength and they are related by $f_{c}=c / \lambda_{\omega}$ At the $m^{\text {th }}$ element, the received signal is given by 


$$
x_{m}(t)=x_{1}(t) \exp \left(-j \frac{2 \pi}{\lambda_{\omega}}(m-1) \frac{d}{c} \sin \left(\theta_{i}\right)\right) \quad i=1,2,3, \ldots, M
$$

Where $x_{l}(t)$ is the received signal vector at the first element of the ULA. The direction vector matrix is given by

$$
A=\left[a\left(\theta_{1}\right), a\left(\theta_{2}\right), \ldots . . a\left(\theta_{N}\right)\right]
$$

Assuming white Gaussian noise $n_{i}(t)$ at all the elements, the received signal at the output of the array is given by,

$$
\begin{aligned}
x(t) & =\left[x_{1}(t), x_{2}(t), x_{3}(t), \ldots . ., x_{M}(t)\right]^{T} \\
& =A S(t)+n(t)
\end{aligned}
$$

Where, $A$ is a $\mathrm{MxN}$ direction or steering vector matrix. If we discretize the above, the input signals of the array are discrete in time and the output of the array is given by

$$
X(k)=A S(k)+n(k) \quad k=1,2,3, \ldots, K
$$

Where, $k$ is the sample instance and $K$ is the number of snapshots. The parameters of the signal from the source which we are interested in are spatial in nature. Hence they require spatial correlation matrix.

$$
\begin{aligned}
& R=E\left\{X(k) X^{H}(k)\right\}=A E\left[S(k) S^{H}(k)\right] A^{H}+\sigma^{2} I \\
& =A R_{S} A^{H}+\sigma^{2} I
\end{aligned}
$$

Where, $R_{s}$ is the signal correlation matrix, $\sigma^{2}$ is the Noise variance and $I$ is the identity matrix.

Practically, the correlation matrix [7] is unknown and it has to be estimated from the array output data. If the underlying processes are ergodic, then the statistical expectation can be replaced by time average.

Let us consider that, $x(k)$ is the signal corrupted by noise having $K$ snapshots are received at the output of the array. The received signal $x(k)$ is denoted by $X$ which is also known as stacked data matrix. The similar stacking is applied to pure signal vector $S(k)$ and the noise vector $n(k)$ as $S$ and $N$ respectively. Equation (9) can be written as

$$
X=A S+N
$$

Where, $X$ is the received noise corrupted signal matrix of size MxK, $A$ is the direction or steering vector matrix of size $\mathrm{MxN}, S$ is the signal matrix of size $\mathrm{NxK}, N$ is the additive white Gaussian matrix of size MxK.

The ensemble correlation matrix estimate is computed by

$$
\hat{R}=\frac{1}{K} \sum_{k=1}^{K} x(k) x^{H}(k)=\frac{1}{K}\left[X X^{H}\right]
$$


Signal \& Image Processing : An International Journal (SIPIJ) Vol.6, No.6, December 2015

\subsection{Subspace based techniques}

The subspace based methods of DOA estimation use the estimated correlation matrix, decomposing it and carrying out the analysis on the decomposition. These techniques were started from a paper published by V.T. Pisarenko [14], later tremendously progressed by the introduction of MUSIC proposed by R.O.Schmidt and followed by ESPRIT by Roy and Kailath. These techniques use the Eigen decomposition of the estimated correlation matrix into signal and noise subspaces.

The array output correlation matrix is given by

$$
\begin{aligned}
\hat{R} & =A E\left[S S^{H}\right] A^{H}+\sigma^{2} I \\
& =A R_{S} A^{H}+\sigma^{2} I
\end{aligned}
$$

Where $R_{s}$ is the signal correlation matrix $\sigma^{2}$ is the noise variance and $I$ is the identity matrix. The correlation matrix of (13) is decomposed using Eigen Value Decomposition (EVD) to obtain

$$
\hat{R}=V \Lambda V^{H}
$$

Where, $V$ is the unitary matrix of Eigen vectors of $R$ as columns, $\Lambda$ is the diagonal matrix of Eigen values of $R$. In the present literature, we assume that the sources are uncorrelated and hence the rank of the correlation matrix $R$ is $\mathrm{M}$ and that of $R_{S}$ is $\mathrm{N}$.

$$
\text { Eigen values of } R: \lambda_{1}>\lambda_{2}>\ldots . .>\lambda_{M}
$$

Eigen values of signal subspace $R_{S}: \lambda_{1}>\lambda_{2}>\ldots . .>\lambda_{\mathrm{N}}$.

Remaining (M-N) Eigen values corresponds to noise subspace. The columns of V are orthogonal. Hence, the correlation matrix can also be decomposed as

$$
\begin{aligned}
\hat{R} & =V \Lambda V^{H} \\
& =V_{S} \Lambda V_{S}{ }^{H}+V_{n} \Lambda_{n} V_{n}{ }^{H}
\end{aligned}
$$

Where $V_{S}$ are signal Eigen vectors, $\Lambda_{S}$ are signal Eigen values both span the signal subspace $E_{S}$. $V_{n}$ and $\Lambda_{n}$ are noise Eigen vectors and Eigen values respectively spanning the complement of signal subspace called noise subspace $E_{n}$.

\subsubsection{MUSIC}

MUSIC Stands for Multiple SIgnal Classification and it is a high resolution DOA estimation algorithm. It gives the estimate of DOA of signals as well as the estimate of the number of signals. In this algorithm, the estimation of DOA can be carried out by using one of the subspaces either noise or signal. The steps followed to estimate DOA using MUSIC are as follows

Step 1: Estimate the correlation Matrix $\hat{R}$ from the equation

$$
\hat{R}=\frac{1}{K}\left[X X^{H}\right]
$$


Signal \& Image Processing : An International Journal (SIPIJ) Vol.6, No.6, December 2015

Step 2: Find the Eigen decomposition of the estimated correlation matrix using the following equation

$$
\hat{R}=\hat{V} \hat{\Lambda} \hat{V}^{H}
$$

Step 3: Find the noise decomposition of $\hat{V}$ matrix to find the span of noise subspace $V_{n}$

Step 4: Plot the MUSIC function $P_{\text {Music }}$ as a function of $\theta$

$$
P_{\text {Music }}=\frac{1}{\mathrm{P} V_{n} a(\theta) \mathrm{P}^{2}}
$$

Step 5: The $M$ Signal directions are the $M$ largest peaks of the plot.

The MUSIC Algorithm along with the required number of operations are summarised in the table below

Table 1: Summary of the MUSIC Algorithm

\begin{tabular}{|l|l|l|}
\hline \multicolumn{2}{|c|}{ Input to the algorithm $\theta, a(\theta)$} \\
\hline No & Operation performed & Complexity \\
\hline 1 & Estimation of Correlation Matrix & $\mathrm{KM}^{2}$ \\
\hline 2 & $\begin{array}{l}\text { Eigen decomposition of correlation Matrix } \\
\text { Selecting the (N-M) Eigen pair to obtain Noise subspace }\end{array}$ & $\mathrm{O}\left(\mathrm{M}^{3}\right)$ \\
\hline 4 & $\begin{array}{l}\text { Plotting the Music function and identifying 'M' Large peaks } \\
\text { of the plot }\end{array}$ & $\left((\mathrm{M}-\mathrm{N}) \mathrm{MK}^{3}\right)$ \\
\hline \multicolumn{2}{|r|}{ Total } & $\mathrm{KM}^{2}+\mathrm{O}\left(\mathrm{M}^{3}\right)+\left((\mathrm{M}-\mathrm{N}) \mathrm{MK}^{3}\right)$ \\
\hline
\end{tabular}

From the Table 1, it has been observed that, the MUSIC algorithm is having the computational complexity of the order of ' $\mathrm{M}^{3}$ '. Search algorithm is needed to decide the largest ' $\mathrm{N}$ ' peaks. This increases the computational complexity.

\subsubsection{Root-MUSIC}

In order to overcome the necessity of comprehensive search algorithm to locate largest ' $\mathrm{N}$ ' peaks in the MUSIC algorithm, a new algorithm which gives the results numerically has been developed. The algorithm is known as Root-MUSIC is a model based parametric estimation technique. It is also a polynomial rooting version of the MUSIC algorithm. The algorithm operates in the following steps.

Step 1: Estimate the Correlation Matrix 'R' from the equation

$$
\hat{R}=\frac{1}{K}\left[X X^{H}\right]
$$

Step 2: Find the Eigen decomposition of the estimated correlation matrix using the following equation 
Signal \& Image Processing : An International Journal (SIPIJ) Vol.6, No.6, December 2015

$$
\hat{R}=\hat{V} \hat{\Lambda} \hat{V}^{H}
$$

Step 3: Find the noise decomposition of ' $V$ ' matrix to find the noise subspace.

Step 4: Find the ' $C$ ' matrix using

$$
C=V_{n} V_{n}^{H}
$$

Step 5: Find ' $\mathrm{C}_{\ell}$ ' by summing the ' $\mathrm{t}^{\mathrm{th}}$ ' diagonal of $C$ using

$$
C_{l}=\sum_{n=m-l} C_{m, n}
$$

Step 6: Find the roots of the resulting polynomial in terms

of (N-1) pairs by

$$
P(Z)=\sum_{-(M-1)}^{(M+1)} C_{l} Z^{l}
$$

Step 7: Of the (N-1) roots within the unit circle, choose the 'M' closest roots to the unit circle.

Step 8: Obtain the DOA using the below formula

$$
\theta=\arcsin \left(\frac{c}{d w} \arg \left(Z_{n}\right)\right)
$$

The operation of the root-MUSIC algorithm along with the number of operations for each important step is summarized in the following table

Table 2: Summary of the operation of Root-MUSIC algorithm

\begin{tabular}{|l|l|l|}
\hline \multicolumn{2}{|c|}{ Input to the algorithm $\theta, a(\theta)$} \\
\hline No & Operation performed & Complexity \\
\hline 1 & Estimation of Correlation Matrix & $\mathrm{KM}^{2}$ \\
\hline 2 & Eigen decomposition of correlation Matrix & $\mathrm{O}\left(\mathrm{M}^{3}\right)$ \\
\hline 3 & Selecting the (N-M) Eigen pair to obtain $V_{n}$ & \multirow{2}{*}{$\mathrm{M}^{2}(\mathrm{M}-\mathrm{N})$} \\
\hline 4 & Finding the $C$ Matrix given by $C=V_{n} V_{n}^{H}$ & $\mathrm{M}$ \\
\hline 5 & Finding $C_{l}=\sum_{n=m-l} C_{m, n}$ & \\
\hline 6 & Finding roots of the polynomial & $8 \mathrm{M}^{2} \mathrm{~K}$ \\
\hline 7 & Calculation of DOA's & \\
\hline & \multicolumn{2}{|c|}{ Total } \\
\hline
\end{tabular}

From Table 2, we can see that the complexity of the operation of the Root-MUSIC algorithm is of the order of ' $\mathrm{M}^{3}$ '. 
Signal \& Image Processing : An International Journal (SIPIJ) Vol.6, No.6, December 2015

\subsubsection{ESPRIT}

The algorithm is based on the rotational invariance property of the signal subspace. Let us define two sub-matrices, $A_{0} \& A_{l}$ by deleting the first and last columns of steering vectors matrix ' $A$ ' respectively. The matrices $A_{0} \& A_{l}$ are related by the following equation

$$
A_{1}=A_{0} \phi
$$

Where, $\phi$ is the diagonal matrix having roots on its diagonal. Here the DOA Estimation problem gets reduced to finding the matrix $\phi$. Considering the correlation matrix $R$ from eq (12), let us obtain a matrix $R_{0}$ which is obtained by deleting last column of $R$ and similarly we obtain a matrix $R$ by deleting last column of $R$.

Let us define two matrices $V_{S O}$ which has ' $\mathrm{N}$ ' largest Eigen vectors of $R_{0}$ as its columns and similarly $V_{S I}$ is a matrix which is having 'N' largest Eigen vectors of $R_{I}$ as its columns. The two matrices $V_{S O}$ and $V_{S I}$ are related by a unique non-singular matrix ' $\psi$ ' given by

$$
V_{S 1}=V_{S 0} \Psi
$$

The same two matrices $V_{S O}$ and $V_{S I}$ are related to the steering vector matrix by a non-singular transformation matrix $\Gamma$ with the help of equations given below

$$
V_{S 0}=A_{0} \Gamma \text { and } V_{S 1}=A_{1} \Gamma=A_{0} \phi \Gamma
$$

Substituting eq(26) in (27) we get

$$
V_{S 0} \Psi=A_{0} \phi \Gamma \text { and } A_{0} \Gamma \Psi=A_{0} \phi \Gamma
$$

$\phi$ is estimated using least square problem, the diagonal elements of $\phi$ are the estimates of the polynomial. The DOA is obtained using (20). The algorithm, along with the number of operations needed is summarized in the table below

Table 3: Summary of operation of ESPRIT Algorithm

\begin{tabular}{|l|l|l|}
\hline \multicolumn{2}{|c|}{ Input to the algorithm $\theta, a(\theta)$} \\
\hline No & Operation performed & Complexity \\
\hline 1 & Estimation of Correlation Matrix & $\mathrm{KM}^{2}$ \\
\hline 2 & Eigen decomposition of correlation Matrix & $\mathrm{O}\left(\mathrm{M}^{3}\right)$ \\
\hline 3 & $\begin{array}{l}\text { inding the signal subspace } \\
\text { orming the } V_{S O} \text { by deleting } 1^{\text {st }} \text { column of } V_{S} \\
\text { orming the } V_{S I} \text { by deleting last column of } V_{S}\end{array}$ & \multirow{2}{*}{$\mathrm{O}\left(\mathrm{N}^{3}\right)$} \\
\hline 4 & Using Least squares solve the resulting equation & \\
\hline 5 & Obtain estimate of MxM Matrix $\psi$ & $\left.\mathrm{KM}^{2}+\mathrm{O}_{(1}\right)+\mathrm{O}\left(\mathrm{N}^{3}\right)$ \\
\hline
\end{tabular}

It has been observed from Table 3 that, the ESPRIT algorithm is having the computational complexity of the order of ' $\mathrm{M}^{3}$ ' as well as ${ }^{\mathrm{N}} \mathrm{N}^{3}$. . By this we can say that this algorithm is computationally most complex of the three algorithms which are considered for the study. 
Signal \& Image Processing : An International Journal (SIPIJ) Vol.6, No.6, December 2015

\subsection{Tracking the DOA}

The Kalman filter (KF) algorithm proposed by R.E.Kalman is considered as the basic of tracking algorithms used in optimum filtering of non-stationary signals. KF algorithm also known as dynamic filtering algorithm is considered as an advantage over the Weiner filter [2] [6] [16] which fail to address the issue of non-stationarity. In DOA tracking we use KF filter to track the optimum DOA estimate. The estimated DOA using one of the procedures above will act as an initial estimate to the Kalman Filter algorithm. Based on the physical model, the algorithm starts tracking the DOA Estimate. The KF algorithm is illustrated using the following steps.

\subsubsection{Tracking model}

Let us consider $\theta_{i}(t), \dot{\theta}_{i}(k), \ddot{\theta}_{i}(k) ; i=1,2,3 \ldots . . q$ gives us the DOA, Angular velocity and angular acceleration of the ' $q$ ' number of sources at time $T$. The equations governing the motion of the $i^{\text {th }}$ source are given by

$$
\left[\begin{array}{c}
\theta_{i}(k+1) \\
\dot{\theta}_{i}(k+1) \\
\ddot{\theta}(k+1)
\end{array}\right]=F\left[\begin{array}{c}
\theta_{i}(k) \\
\dot{\theta}_{i}(k) \\
\ddot{\theta}(k)
\end{array}\right]+\left[\begin{array}{c}
\omega_{i 1}(k) \\
\omega_{i 2}(k) \\
\omega_{i 3}(k)
\end{array}\right]
$$

Where Matrix $F$ is given by

$$
F=\left[\left(\begin{array}{ccc}
1 & T & \frac{T^{2}}{2} \\
0 & 1 & T \\
0 & 0 & 1
\end{array}\right)\right]
$$

Where $T$ is the sampling duration and $\omega_{i}(k), i=1,2,3 \ldots . q$ are random process noise responsible for the random disturbances. It is assumed that $\omega_{i}(k)$ is zero mean white Gaussian noise with covariances indicated as follows

$$
Q_{i}=E\left[\omega_{i}(k) \omega_{i}^{T}(k)\right]
$$

In the tracking model illustrated above, we assume that the acceleration remains constant throughout the sampling interval.

\subsubsection{Tracking Algorithm}

In the present study, single source is being tracked. The tracking algorithm is illustrated as follows $x_{i}(k)$ is the state of the ' $q$ ' sources at ' $k$ ' and is given by 
Signal \& Image Processing : An International Journal (SIPIJ) Vol.6, No.6, December 2015

$$
x_{i}(k)=\left[\begin{array}{c}
\theta_{i}(k) \\
\dot{\theta}_{i}(k) \\
\ddot{\theta}(k)
\end{array}\right] \quad i=1,2, \ldots, q
$$

Using equation (29) the source motion governing model, we can write (30) as

$$
x_{i}(k+1)=F x_{i}(k)+\omega_{i}(k)
$$

$\hat{\theta}_{i}(k)$ is the optimum DOA Estimate of $\theta_{i}(k)$ based on the data obtained during the interval $[(k T,(k+1) T]$. Based on this we can write the measurement equation as

$$
\hat{\theta}_{i}(k)=\theta_{i}(k)+\eta_{i}(k) \quad i=1,2,3, \ldots, q
$$

using equations (34) and considering the optimum DOA Estimate, a Kalman filter is used to track the source's state estimate. The state estimation is carried out using the following components.

We can rewrite the equation (34) as

$$
\hat{\theta}_{i}(n)=h\left[\begin{array}{c}
\theta_{i}(n) \\
\dot{\theta}_{i}(n) \\
\ddot{\theta}_{i}(n)
\end{array}\right]+\eta_{i}(n) \quad i=1,2,3, \ldots, q
$$

Where $h=[1,0,0]$ since we are going to track only angular position, we neglect the angular velocities and acceleration and hence the ' $h$ ' vector. Using Equations (32-35) the Kalman filter equation can be written as

$$
\left[\begin{array}{c}
\hat{\theta}_{i}(n \mid n) \\
\dot{\theta}_{i}(n \mid n) \\
\ddot{\theta}_{i}(n \mid n)
\end{array}\right]=\left[\begin{array}{c}
\hat{\theta}_{i}(n \mid n-1) \\
\hat{\theta}_{i}(n \mid n-1) \\
\hat{\theta}_{i}(n \mid n-1)
\end{array}\right]+L_{i}(n)\left[\hat{\theta}_{i}(n)-\hat{\theta}_{i}(n \mid n-1)\right]
$$

The first term in the RHS of (36) are predicted estimates, the prediction is carried out using the measurements up to $(n-1) T$.

The predicted state estimates of $\hat{\theta}_{i}(n \mid n), \dot{\theta}_{i}(n \mid n), \ddot{\theta}_{i}(n \mid n)$ are given by

$\left[\hat{\theta}_{i}(n \mid n-1), \hat{\dot{\theta}}_{i}(n \mid n-1), \hat{\ddot{\theta}}_{i}(n \mid n-1)\right]$ respectively. The Kalman gain $L_{i}(n)$ acts as a weighted compensator is given by

$$
L_{i}(n)=\frac{P_{i}(n \mid n-1) h^{T}}{h P(n \mid n-1) h^{T}+J_{i i}^{-1}}
$$

Where, $J_{i i}$ is the $i^{\text {th }}$ element of the Fisher information Matrix.

The Kalman filter recursions are carried out in the following steps

1. In the first interval, one of the optimum procedures to obtain DOA is used to find the initial estimate of DOA. 
Signal \& Image Processing : An International Journal (SIPIJ) Vol.6, No.6, December 2015

2. In the next step we use the optimum DOA estimate as the initial value and start tracking the DOA Estimates. The Kalman filter algorithm is summarised in Table 4

Table4: Summary of KF Algorithm

\begin{tabular}{|l|l|}
\hline No & Operation performed \\
\hline Step 1 & Initialization of the KF Algorithm \\
\hline Step 2 & $\begin{array}{l}\text { Time recursion for } n=1,2,3, \ldots \ldots . \\
\text { a)Finding the signal prediction } \\
\text { b) Finding the data prediction } \\
\text { c) Determination of A priori error covariance } \\
\text { d) Finding the Kalman Gain } \\
\text { e) Finding the Signal Update } \\
\text { f)Determination of A posteriori error covariance }\end{array}$ \\
\hline Step 3 & \begin{tabular}{l} 
Obtaining the filtered estimate ( Output) \\
\hline
\end{tabular} \\
\hline
\end{tabular}

\subsection{Results and Discussions}

In this section, in order to demonstrate the numerical properties, convergence characteristics and direction finding abilities of the spectral based technique MUSIC and parametric techniques Root-MUSIC,ESPRIT have been compared for different cases to decide optimum among them using several simulations. Later, the process of comparison is continued by providing DOA estimates to the Kalman filter tracking algorithm, where we observe which technique makes the best estimate for the purpose of tracking. Fig 2 shows the Antenna Beam pattern for DOA Estimation using MUSIC Algorithm. In the present simulation, three non-coherent, narrowband sources of equal power are impinging from the broadside of the array with noise power $25 \mathrm{~dB}$ SNR from the directions $-20^{\circ}, 20^{\circ}, 40^{\circ}$. The simulation is carried out for 50 runs with 50 snapshots.

Fig 3 and Fig 4 shows the Histogram plot of Root-MUSIC, ESPRIT algorithm respectively. The simulations carried out for three non-coherent, narrowband sources of equal power are impinging from the broadside of the array with noise power $25 \mathrm{~dB}$ SNR from the directions $-20^{\circ}, 20^{\circ}$, $40^{\circ}$.The simulation is carried out for 50 runs with 50 snapshots. These algorithms give the values of the estimated parameter in terms of numbers directly.

Table 5 shows that, all the algorithms work at less number of array elements. Since we use three sources, four array elements are sufficient to estimate the DOA. However, as we increase the number of array elements there is no significant change in estimation accuracy. Increase in the number of array elements increases the computational complexity due to the increase in the size of the correlation matrix and hence the cost. 
Signal \& Image Processing : An International Journal (SIPIJ) Vol.6, No.6, December 2015

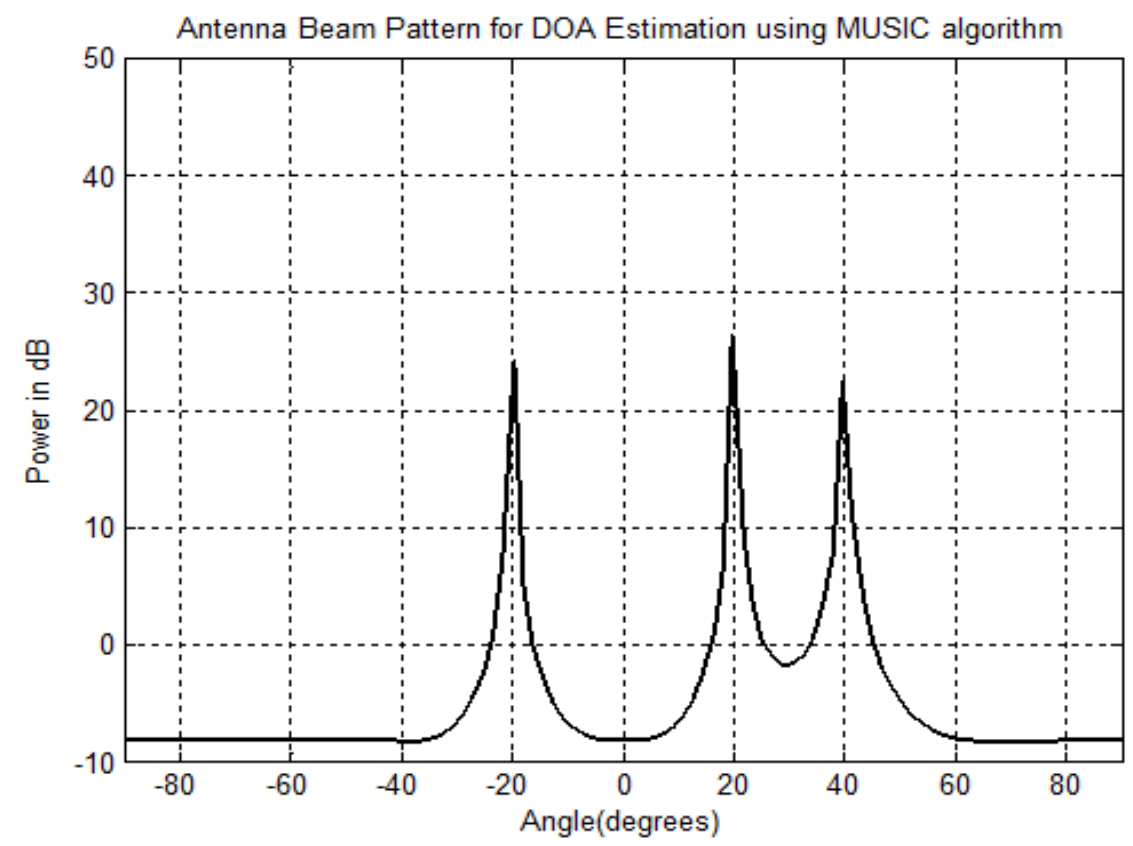

Fig 2 : Antenna beam Pattern for DOA Estimation using MUSIC algorithm (Number of antenna elements $=7, \mathrm{SNR}=25 \mathrm{~dB}$,Number of snapshots $=50$ )

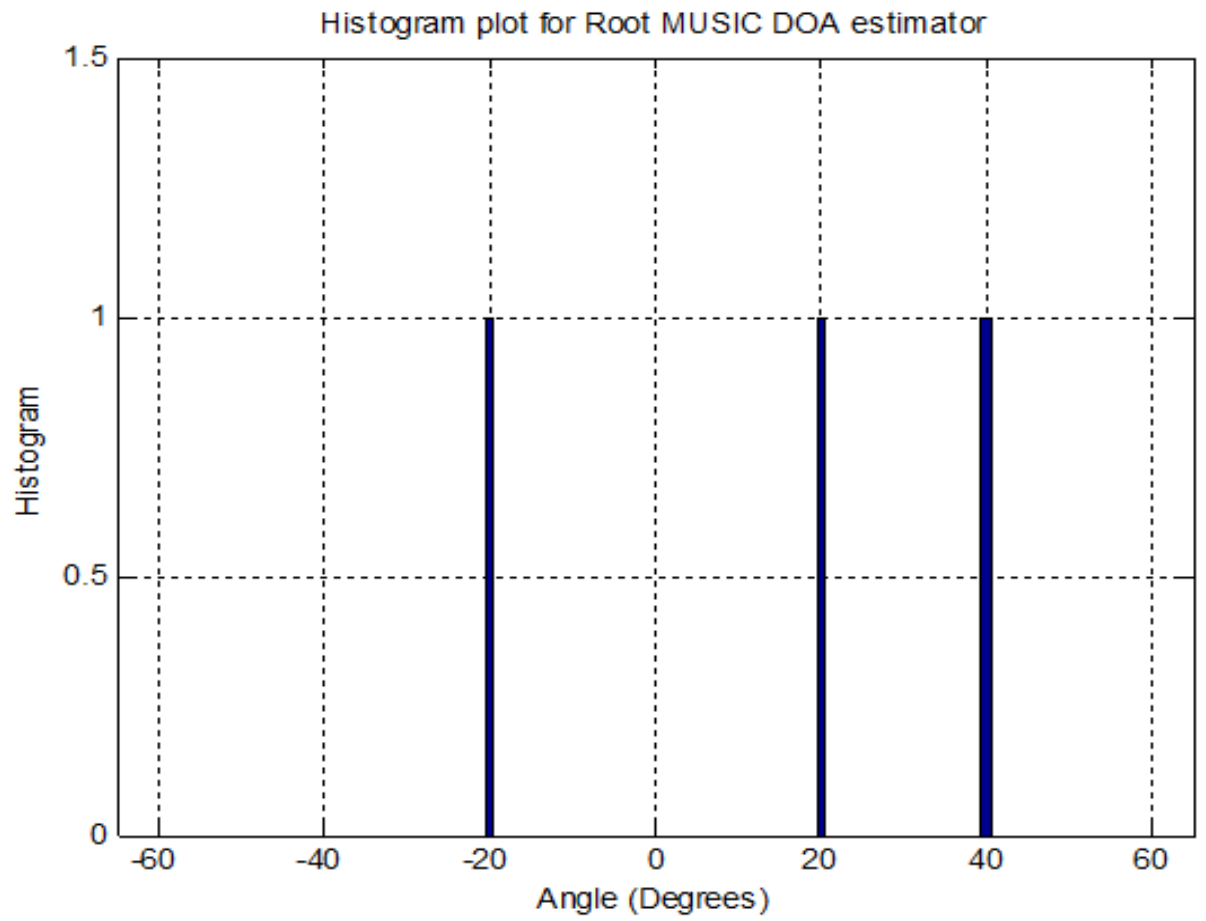

Fig 3 : Histogram plot for DOA Estimation using Root-MUSIC algorithm (Number of antenna elements $=7, \mathrm{SNR}=25 \mathrm{~dB}$, Number of snapshots $=50$ ) 
Signal \& Image Processing : An International Journal (SIPIJ) Vol.6, No.6, December 2015

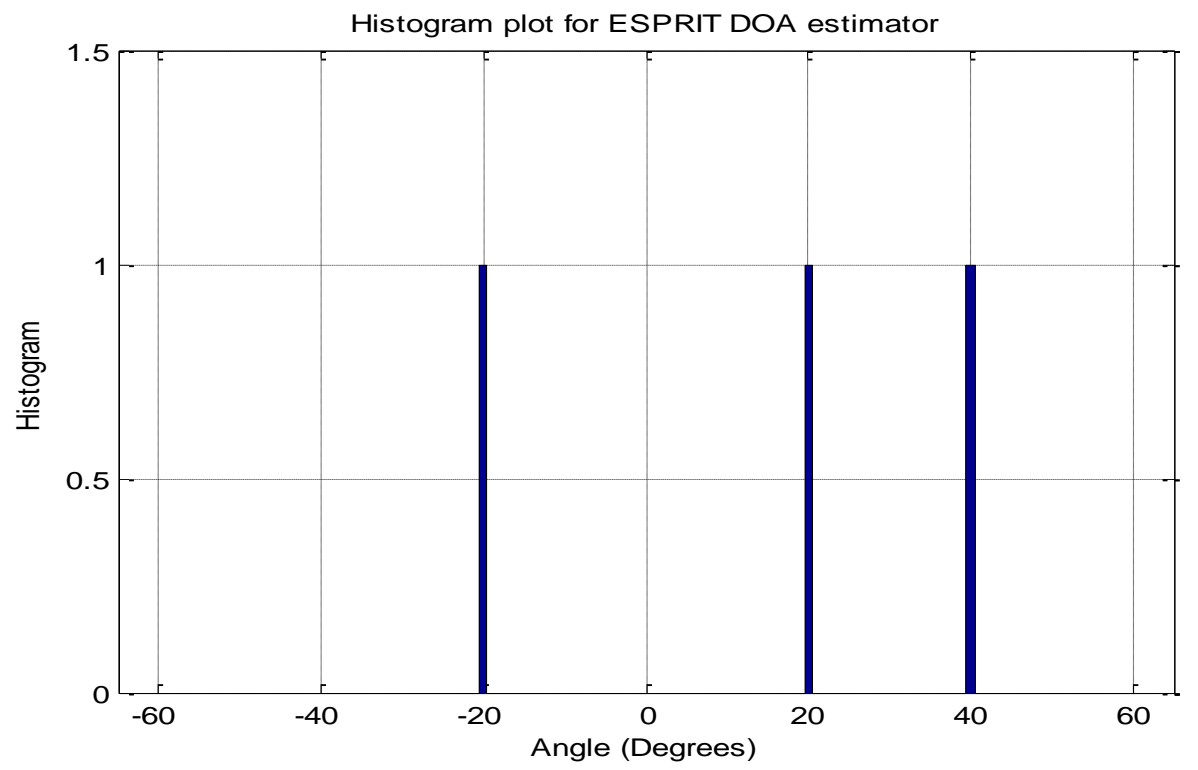

Fig 4: Histogram plot for DOA Estimation using ESPRIT algorithm (Number of antenna elements $=7, \mathrm{SNR}=25 \mathrm{~dB}$, Number of snapshots $=50$ )

Table 5: Performance of the Algorithms for varying number of array elements

\begin{tabular}{|c|c|c|c|c|c|c|c|c|c|}
\hline \multirow{3}{*}{\begin{tabular}{c|} 
No of \\
array \\
elements
\end{tabular}} & \multicolumn{9}{|c|}{ SNR $=25 \mathrm{~dB}$, Snapshots $=50$, Sources are at $\left\{-20^{\circ}, 20^{\circ}, 40^{\circ}\right\}$} \\
\hline & \multicolumn{3}{|c|}{ MUSIC } & \multicolumn{3}{|c|}{ Root-MUSIC } & \multicolumn{3}{|c|}{ ESPRIT } \\
\hline & $-20^{\circ}$ & $20^{\circ}$ & $40^{\circ}$ & $-20^{\circ}$ & $20^{\circ}$ & $40^{\circ}$ & $-20^{\circ}$ & $20^{\circ}$ & $40^{\circ}$ \\
\hline 7 & -19.91 & 19.89 & 39.86 & -19.9925 & 19.9744 & 39.9865 & -20.0208 & 19.9767 & 39.9757 \\
\hline 21 & -19.90 & 19.90 & 39.87 & -19.9955 & 19.9948 & 39.9917 & -19.9969 & 19.9909 & 39.9956 \\
\hline 50 & -19.89 & 19.86 & 39.77 & -19.9992 & 19.9999 & 39.9995 & -20.0021 & 19.9991 & 40.0026 \\
\hline 100 & -19.51 & 19.53 & 39.70 & -20.0001 & 20.0003 & 39.9994 & -20.0001 & 20.0009 & 39.9980 \\
\hline 200 & -19.46 & $\begin{array}{l}19.43 \\
\end{array}$ & 39.37 & -20.0002 & 20.0001 & 40.0002 & -20.0017 & $\begin{array}{l}19.9989 \\
\end{array}$ & 40.0006 \\
\hline
\end{tabular}

Table 6: Performance of the Algorithms for varying number of snapshots

\begin{tabular}{|c|c|c|c|c|c|c|c|c|c|}
\hline \multirow{3}{*}{$\begin{array}{c}\text { No of } \\
\text { snapshots }\end{array}$} & \multicolumn{9}{|c|}{ SNR $=25 \mathrm{~dB}$, Number of array elements $=7$, Sources are at $\left\{-20^{\circ}, 20^{\circ}, 40^{\circ}\right\}$} \\
\hline & \multicolumn{3}{|c|}{ MUSIC } & \multicolumn{3}{|c|}{ Root-MUSIC } & \multicolumn{3}{|c|}{ ESPRIT } \\
\hline & $-20^{\circ}$ & $20^{\circ}$ & $40^{\circ}$ & $-20^{\circ}$ & $20^{\circ}$ & $40^{\circ}$ & $-20^{\circ}$ & $20^{\circ}$ & $40^{\circ}$ \\
\hline 1 & -13.12 & 12.8 & 31.2 & -28.25 & 15.69 & 29.13 & -28.99 & 12.7046 & 36.939 \\
\hline 10 & -18.91 & 18.2 & 39.8 & -19.98 & 20.127 & 39.89 & -19.94 & 20.105 & 39.941 \\
\hline 20 & -19.89 & 19.97 & 39.92 & -19.98 & 19.9897 & 39.968 & -20.0057 & 20.0052 & 39.9982 \\
\hline 40 & -19.92 & 19.89 & 39.87 & -20.037 & 20.0144 & 40.022 & -20.0295 & 20.0010 & 39.9978 \\
\hline 100 & -19.98 & 19.96 & 39.93 & -20.0096 & 19.9901 & 40.0182 & -20.0062 & 20.0211 & 40.0037 \\
\hline 200 & -19.94 & 19.93 & 39.82 & -20.0018 & 20.0022 & 40.0128 & -20.0037 & 19.9993 & 40.0031 \\
\hline 500 & -19.95 & 19.95 & 39.93 & -20.0006 & 19.9970 & 40.0071 & -19.9977 & 20.0022 & 40.0163 \\
\hline 1000 & -19.96 & 19.97 & 39.96 & -20.0002 & 20.0107 & 40.0040 & -20.0013 & 19.9929 & 40.0054 \\
\hline
\end{tabular}

Table 6 shows the performance of the DOA Estimation algorithms for varying number of snapshots. At single snapshot, all the algorithms won't perform accurately. As the number of snapshots gets increased to 10, 20 and further, the accuracy in the estimation increases substantially and hence the computational complexity. However, Root-MUSIC and ESPRIT algorithms perform much better. Of the two (Root-MUSIC and ESPRIT) algorithms, ESPRIT algorithm performs best even at 10 snapshots. 
Signal \& Image Processing : An International Journal (SIPIJ) Vol.6, No.6, December 2015

Table 7: Performance of the Algorithms for varying SNR

\begin{tabular}{|c|c|c|c|c|c|c|c|c|c|}
\hline \multirow{3}{*}{$\begin{array}{l}\text { SNR } \\
\text { (dB) }\end{array}$} & \multicolumn{9}{|c|}{ Number of snapshots $=50$, Number of array elements $=7$, Sources are at $\left\{-20^{\circ}, 20^{\circ}, 40^{\circ}\right\}$} \\
\hline & \multicolumn{3}{|c|}{ MUSIC } & \multicolumn{3}{|c|}{ Root-MUSIC } & \multicolumn{3}{|c|}{ ESPRIT } \\
\hline & $-20^{\circ}$ & $2 \mathbf{2 0}^{\mathbf{0}}$ & $40^{\circ}$ & $-20^{\circ}$ & $20^{\circ}$ & $40^{\circ}$ & $-20^{\circ}$ & $20^{\circ}$ & $40^{\circ}$ \\
\hline 1 & -19.12 & 21.2 & 38.3 & -19.8053 & 19.8053 & 39.1318 & -20.5167 & 19.9382 & 39.9006 \\
\hline 2 & -20.91 & 19.1 & 39.4 & -19.2629 & 19.9044 & 39.6671 & -19.3558 & 19.9956 & 39.7850 \\
\hline 5 & 20.43 & 10 & 40.25 & -20.1 & & & 967 & 19.1323 & 39.9528 \\
\hline 10 & -19.18 & 19.63 & 39.65 & -19.9368 & 19.812 & 39.9518 & -19.9845 & 19.9206 & 39.9025 \\
\hline 25 & -19.89 & 19.88 & 40.23 & -19.9976 & 19.9751 & 40.0148 & -20.0001 & 19.9868 & 39.9958 \\
\hline 50 & -19.81 & 19.86 & 39.87 & -19.9998 & 20.0001 & 39.9994 & -20.0037 & 19.9988 & 40.0007 \\
\hline 80 & -19.91 & 19. & 39.93 & -20.0001 & & 40.1 & -20 & 20.0004 & 39.9999 \\
\hline 90 & -19.94 & 19.99 & 39.99 & -20.000 & 20.0000 & 40.0000 & -20.0000 & 20.0000 & 40.0000 \\
\hline
\end{tabular}

Table 7 shows the performance of the DOA Estimation algorithms for varying SNR. At low SNR, the accuracy of MUSIC algorithms is not good whereas the Root-MUSIC, ESPRIT algorithms perform relatively well. As increase SNR, the accuracy in the estimation increases in the case of MUSIC algorithm. However, Root-MUSIC and ESPRIT algorithms perform much better even at low SNR. Of the two (Root-MUSIC, ESPRIT) algorithms, ESPRIT performs much better.

Later, a single moving source is considered and its estimated DOA is taken as an initial value and is provided to the Kalman filter for the purpose of tracking. Here, $10^{\circ}$ initial value is assumed, the estimated DOA by all the three algorithms is given to Kalman filter model for tracking and the performance is analysed for each algorithm.

The Figs 5,6 and 7depict the performance of Kalman filter algorithm for the initial value estimated using MUSIC, Root-MUSIC, and ESPRIT algorithms respectively. The KF tracking algorithm is able to track the source whose initial value is estimated by all the DOA Estimation algorithms from $10^{\circ}$ to $170^{\circ}$. Of the three algorithms considered above, tracking the initial estimate using ESPRIT algorithm is found to be better compared to other two techniques.

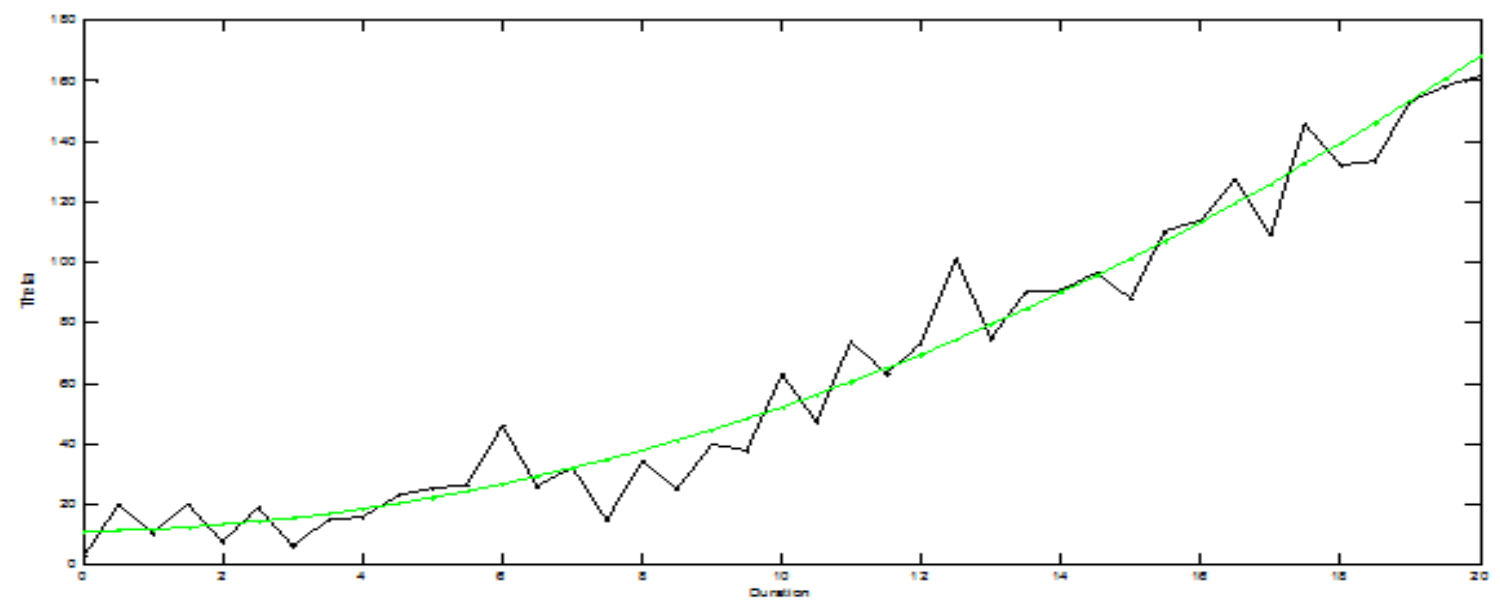

Fig 5: Tracking the DOA Estimation ( Initial Value MUSIC) using Kalman Filter. 
Signal \& Image Processing : An International Journal (SIPIJ) Vol.6, No.6, December 2015

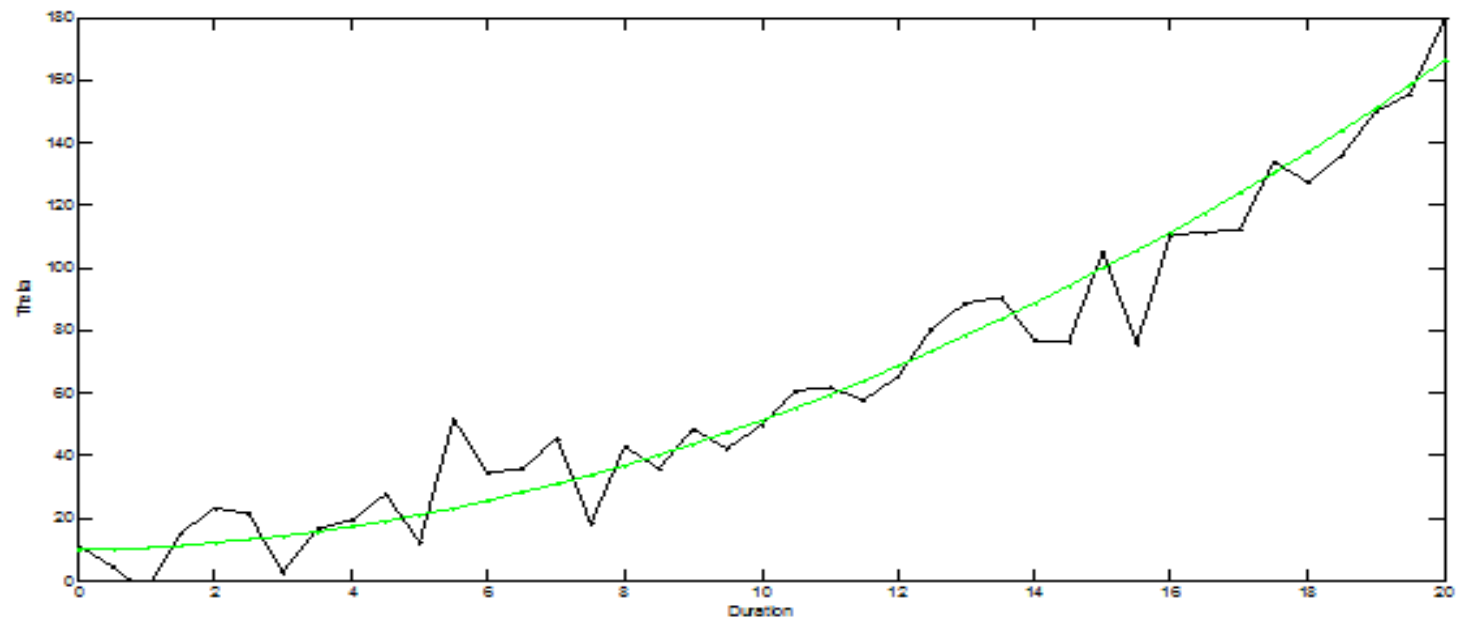

Fig 6: Tracking the DOA Estimation (Initial Value Root-MUSIC) using Kalman Filter.

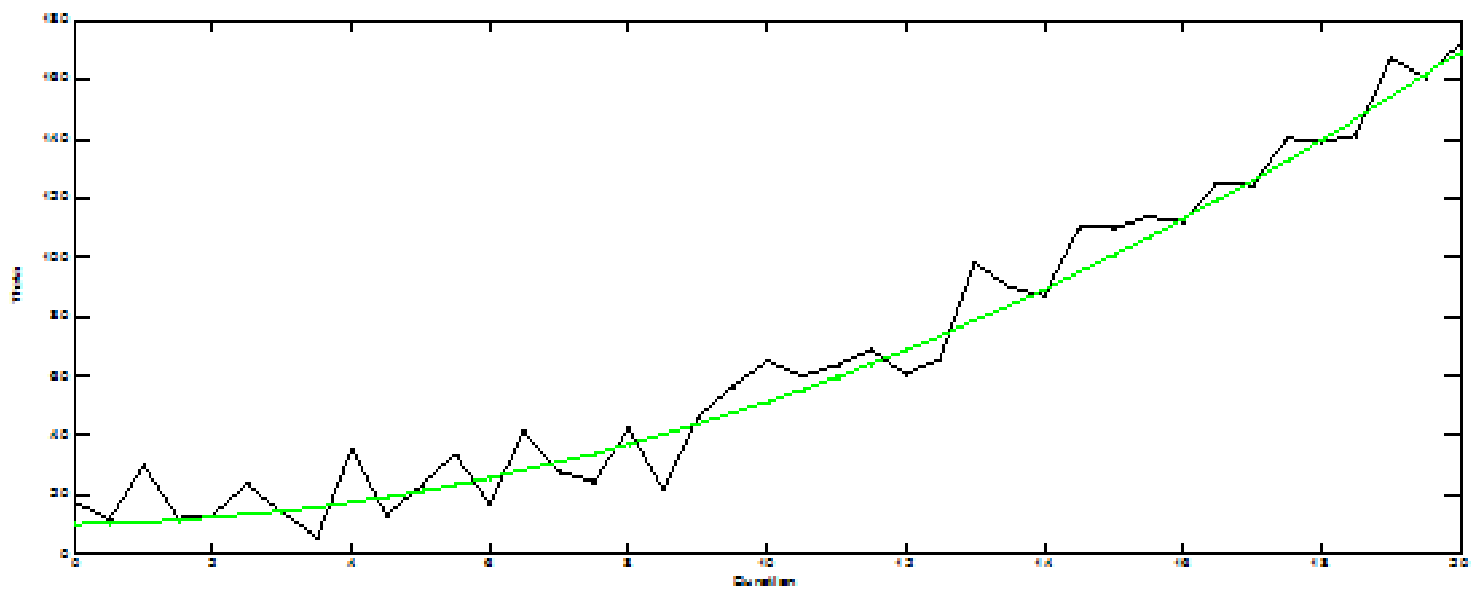

Fig 7: Tracking the DOA Estimation (Initial Value ESPRIT) using Kalman Filter.

\subsection{Conclusions and future work}

The present study depicts that, all the three DOA Estimation algorithms are capable of estimating the DOA and amicably work with Kalman filter algorithm to track the moving source. However, ESPRIT algorithm being computationally complex is highly accurate and acts as best initial estimate to the Kalman filter tracking algorithm. ESPRIT, can be used if abundant computational resources are available. Suitable modifications such as TLS [15]-ESPRIT can be an alternative to ESPRIT. A Comparative study of DOA Estimation and Tracking of Multiple, Coherent and Wideband sources will be addressed in further communication.

\section{REFERENCES}

[1] K. Hamid and M. Viberg, "Two decades of array signal processing research," IEEE Signal Process. Mag., vol. 13, no. 4, pp. 67-94, 1996.

[2] S Haykin Adaptive Filter Theory, 5 edition. Upper Saddle River, New Jersey: Prentice Hall, 2013. 
Signal \& Image Processing : An International Journal (SIPIJ) Vol.6, No.6, December 2015

[3] R. O. Schmidt, "Multiple emitter location and signal parameter estimation," Antennas Propag. IEEE Trans. On, vol. 34, no. 3, pp. 276-280, 1986.

[4] B. Friedlander, "The root-MUSIC algorithm for direction finding with interpolated arrays," Signal Process., vol. 30, no. 1, pp. 15-29, 1993.

[5] R. Roy and T. Kailath, "ESPRIT-estimation of signal parameters via rotational invariance techniques,” Acoust. Speech Signal Process. IEEE Trans. On, vol. 37, no. 7, pp. 984-995, 1989.

[6] M. H. Hayes, Statistical digital signal processing and modeling. John Wiley \& Sons, 2009.

[7] G. H. Golub and C. F. Van Loan, Matrix computations, vol. 3. JHU Press, 2012.

[8] Jagadeesha S. N. "A Comparative Study of Adaptive Algorithms with Applications toBeamforming." $\mathrm{PhD}$ thesis, Department of Electronics and Computer Engineering, University of Roorkee, Roorkee, India, October 1994.

[9] Yerriswamy.T, "Direction of arrival estimation using array signal processing techniques" $\mathrm{PhD}$ thesis, Faculty of computer science and engineering, VTU, India, August 2012.

[10] A B Greshman,Sergiy AV and K M Wong. Maximum likelihood direction of arrival estimation in unknown noise fields using sparse sensor arrays. IEEE Trans on signal processing 53(1):34-43,2005

[11] Lal C. Godara. "Application of antenna array to mobile communications - part i: Performance improvement, feasibility, and system considerations." IEEE Proc, Vol 85 no7 pp 1031-1060, 1997.

[12] J. Capon. High resolution frequency-wavenumber spectrum analysis. In IEEE Proc,Vol 57,Pages $1408-1418,1969$

[13] Satish chandran Advances in Direction of Arrival Estimation Artech House,London,2010

[14] V.T.Pisarenko. The retrieval of harmonics from a covariance function. Geophysics J Royal Astro, Sec, 33(3):347-366,1973

[15] Gene H Golub and C F Van loan. An analysis of the total least squares problem.SIAM,17(6):883893,1980

[16] R.E.Kalman A new approach to linear filtering and prediction problems Journal of Basic engineering 82(1): $35-45$

\section{AUTHORS}

VenuMadhava.M received his B.E. ,in Instrumentation Technology and M.Tech., in Industrial Electronics, from Visvesvaraya Technological University, Belgaum, Karnataka India in 2004 and 2009 respectively. He is currently working towards his Doctoral Degree from Visvesvaraya Technological University, Belgaum, Karnataka, India. At present he is working as Assistant Professor, in the Department of Instrumentation Technology,Proudhadevaraya Institute of Technology,(Affiliated to Visvesvaraya Technological University), Hospet, Karnataka, India.

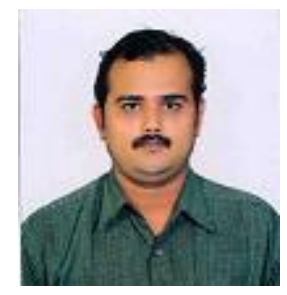


Signal \& Image Processing : An International Journal (SIPIJ) Vol.6, No.6, December 2015

Dr. S. N. Jagadeesha received his B.E., in Electronics and Communication Engineering, from University B. D. T College of Engineering., Davangere affiliated to Mysore University, Karnataka, India in 1979, M..E. from Indian Institute of Science (IISC), Bangalore, India specializing in Electrical Communication Engineering., in 1987 and Ph.D. in Electronics and Computer Engineering., from University of Roorkee (I.I.T, Roorkee), Roorkee, India in 1996. He is an IEEE member and Fellow, IETE. His research interest includes Array Signal Processing, Wireless Sensor Networks and Mobile Communications. He has published and presented many papers

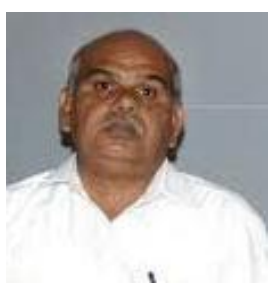
on Adaptive Array Signal Processing and Direction-of-Arrival estimation. Currently he is Professor in the Department of Computer Science and Engineering, Jawaharlal Nehru National College of Engg. (Affiliated to Visvesvaraya Technological University), Shimoga, Karnataka, India.

Dr.Yerriswamy received his B.E. in Electronics and Communication Engineering, From RYMEC, Bellary affiliated to Gulbarga University, Gulbarga, Karnataka, India in 2000,M.Tech in Network and Internet Engineering from JNNCE, Shimoga, Affiliated to Visvesvaraya Technological University, Belgaum, India in 2005 and $\mathrm{Ph} . \mathrm{D}$ in the Faculty of Computer and Information Science form Visvesvaraya Technological University , Belgaum, Karnataka, India He is a member of ISTE. His research interests includes Array Signal Processing, Wireless Sensor Networks, Cognitive Radios. He has published many papers in Direction-of-Arrival estimation

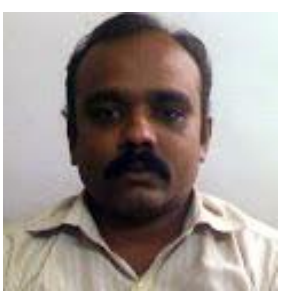
and Array signal processing. Currently he is Professor in the Department of Computer science and Engineering, KLE Institute of Technology, (Affiliated to Visvesvaraya Technological University, Belgaum), Hubli, Karnataka, India 